\title{
First experimental check of the GDH sum rule
}

\author{
Günter Zeitler* for the GDH-Collaboration \\ Physikalisches Institut IV, Universität Erlangen-Nürnberg, Erwin-Rommel-Str. 1, \\ 91058 Erlangen, Germany \\ E-mail: 'guenter.zeitler@physik.uni-erlangen.de!
}

ABstRACT: The GDH-Collaboration checks the very fundamental GDH sum rule experimentally for the first time. We measure the helicity dependent total photoabsorption cross section with circularly polarized real photons and longitudinally polarized nucleons in the photon energy range 0.14-3.1 GeV at tagged photon facilities at MAMI (Mainz) and ELSA (Bonn). Our new data taken at ELSA up to $1.9 \mathrm{GeV}$ for the proton give access to the energy behavior of the GDH integrand and of the running GDH integral.

\section{Introduction}

The GDH sum rule was derived in 1966 by Gerasimov, and independently by Drell and Hearn [i.1]. It connects well known static properties (relative precision better than $10^{-6}$ ) of the nucleon, like the anomalous magnetic moment $\kappa$ and the mass $m$, to dynamic observables of the nucleon. As an observable of the dynamics of the excitation spectrum, the total cross section $\sigma$ of circularly polarized real photons on longitudinally polarized nucleons is regarded in the helicity states $3 / 2$ (spins parallel) and $1 / 2$ (spins antiparallel). Its difference is weighted by the inverse of the photon energy $\nu$ and integrated up to infinity ( $\alpha$ denotes the fine-structure constant):

$$
\int_{0}^{\infty} d \nu \frac{\sigma_{3 / 2}(\nu)-\sigma_{1 / 2}(\nu)}{\nu}=\frac{2 \pi^{2} \alpha}{m^{2}} \kappa^{2}
$$

Its dispersion theoretic derivation is based on very fundamental physical principles: Lorentz and gauge invariance allow to express the Compton forward amplitude by a spindependent and a spin-independent amplitude. Unitarity of the scattering amplitude leads to the optical theorem, which relates the forward amplitude to the total photoabsorption cross section. Due to causality the Compton forward amplitude is analytic and by assuming that no subtraction is necessary (no-subtraction-hypothesis) one can derive a dispersion

\footnotetext{
${ }^{*}$ Speaker.
} 
relation which can be Taylor expanded. Lorentz and gauge invariance are again the underlying principles of the Low-Theorem [2i], which holds for very low photon energies. This theorem connects the spin-dependent scattering amplitude in first order with $\kappa$ and $m$ and in third order of energy with the spin polarizability $\gamma_{0}$. If one compares this expression in first order of energy with the Taylor expanded dispersion relation, also in first order of energy, one directly ends up with the GDH sum rule (Eq. ( (i) (i)

The experimental verification of the sum rule is most likely a validation of the nosubtraction-hypothesis since this is the only non-fundamental part of the derivation. Additionally, the energy dependence of the cross section in the two helicity states gives important constraints for multipole analyses in the resonance region and makes restrictions about the validity of Regge models.

\section{Experimental setup}

The GDH-experiment is carried out at two accelerators to cover a wide photon energy range. At MAMI we measure with the cylindrical $94 \% \cdot 4 \pi$-detector DAPHNE in the resonance region from pion threshold to $0.8 \mathrm{GeV}$. Data taking on the proton was finished

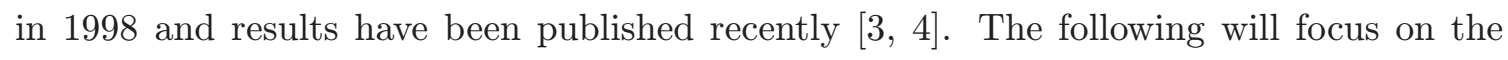
experimental setup at the electron stretcher accelerator facility ELSA in Bonn and on our new results obtained there in the energy range from $0.7-1.9 \mathrm{GeV}$.

\subsection{Circularly polarized photon beam}

Polarized electrons, which are produced by photo effect of a strained superlattice GaAscrystal, are accelerated in ELSA up to $3.2 \mathrm{GeV}$ and are finally extracted to the experiment with a duty cycle of up to $95 \%$. Since ELSA is a circular accelerator, depolarizing resonances occur which have to be overcome $\left[\begin{array}{c}{[\overline{5}} \\ -1\end{array}, \overline{\underline{6}}\right]$. In addition, the vertically orientated electron spin has to be rotated in the external beam line to the longitudinal direction for the experiment. Thus, the polarization of the delivered electrons depends on energy and is typically $50-75 \%$ at a maximum extracted beam current of $2 \mathrm{nA}$. The electron beam helicity is randomly reversed at the source every few seconds both to gain access to a measurement in the two helicity states and to avoid systematic errors.

Circularly polarized photons are produced by bremsstrahlung from longitudinally polarized electrons in a thin metal radiator foil. The helicity transfer depends only on the ratio of the energies of the emitted photon and of the primary electron $[\bar{i}]$. The tagging system covers an energy range of $68-96 \%$ of the primary electron energy. Consequently more than $80 \%$ of the electron polarization is transferred to the photon and 5 primary electron energy settings $(1.0,1.4,1.9,2.4,3.2 \mathrm{GeV})$ are used to cover the photon energy range at ELSA (0.7-3.1 GeV).

An active collimator system [i్ inate low energy photon background produced by the collimation process and to determine the photon flux correctly even at a tagged bremsstrahlung rate of typically $1.3 \mathrm{MHz}$.

The electron polarization is permanently monitored by the GDH-Møller-Polarimeter situated in the primary electron beam behind the tagging system. The Møller-target con- 
sists of magnetized Vacoflux foils, which provide polarized electrons for the Møller scattering process. Both scattered electrons are momentum separated by a dipole magnet and are detected in coincidence in an arrangement of 14 leadglass detectors. This two-arm spectrometer has an acceptance of $\Theta_{C M S}=\left[65^{\circ}, 115^{\circ}\right]$ and allows a fast diagnostics of the electron beam polarization with almost no background contamination.

\subsection{Longitudinally polarized target}

The actively collimated photon beam impinges on the longitudinally polarized target nuclei which are provided within a horizontal ${ }^{3} \mathrm{He} /{ }^{4} \mathrm{He}$ dilution cryostat $[1 \overline{1} 0.1]$. This cryostat is operated in the frozen-spin mode at about $60 \mathrm{mK}$. Beads of butanol $\left(\mathrm{H}\left(\mathrm{CH}_{2}\right)_{4} \mathrm{OH}\right)$ endowed with paramagnetic radicals are used as target material. This material can be polarized by the dynamic nuclear polarization technique up to $85 \%$. Since C and O nuclei are spinless particles only the $\mathrm{H}$ is polarized. By using an internal superconducting holding coil with a magnetic holding field of $0.4 \mathrm{~T}$ we achieved decay times of 200 hours. In particular, the internal holding coil has the advantage of generating only a low fringe field in the GDH-Detector. The target was repolarized approximately every two days. Usually the spin orientation was reversed on that occasion to allow for systematic studies. The target polarization is measured by an NMR-system before and after re-polarization. Due to its horizontal design the cryostat can be inserted into the GDH-Detector from upstream side, resulting in a minimal dead solid angle.

\subsection{GDH-Detector}

In the ELSA photon energy range, photoabsorption processes lead to multi particle final states which are hard to detect individually. In addition, some of these partial channels are even unknown. To avoid systematic uncertainties arising from unobserved final states the total photoabsorption cross section is measured inclusively.

The concept of the GDH-Detector is is that at least one reaction product is detected from all possible hadronic final states with almost complete acceptance concerning solid angle and efficiency. These conditions are fulfilled by an arrangement of 7 hadron detection modules surrounding the target with a solid angle of $99.6 \% \cdot 4 \pi$. Each module consists of a scintillator plate for detecting charged hadrons and a following lead-scintillator sandwich for detecting decay photons. The detection efficiency for the hadronic final states is higher than $99 \%$. An important part of the detector concept is the suppression of electromagnetic background caused by pair production in the field of nuclei and by Compton scattering off orbital electrons. Due to the Lorentzboost this background is strongly emitted into forward directions. With the help of a threshold $\mathrm{CO}_{2}$-Čerenkov detector, which covers polar angles of $0^{\circ} \leq \theta \leq 15^{\circ}$, these particles can be detected and suppressed with an efficiency of (99.990 $\pm 0.003) \%$.

The complete setup has been tested extensively with unpolarized beam and nuclei. We obtained unprecedented data quality for solid state targets like Be and C. An important point was to prove that the GDH-Detector is able to measure cross section differences. From the measured cross section of a solid state $\left[\mathrm{CH}_{2}\right]_{n}$-target we subtracted the C-cross section to determine the H-cross section. The good agreement with literature data shows 


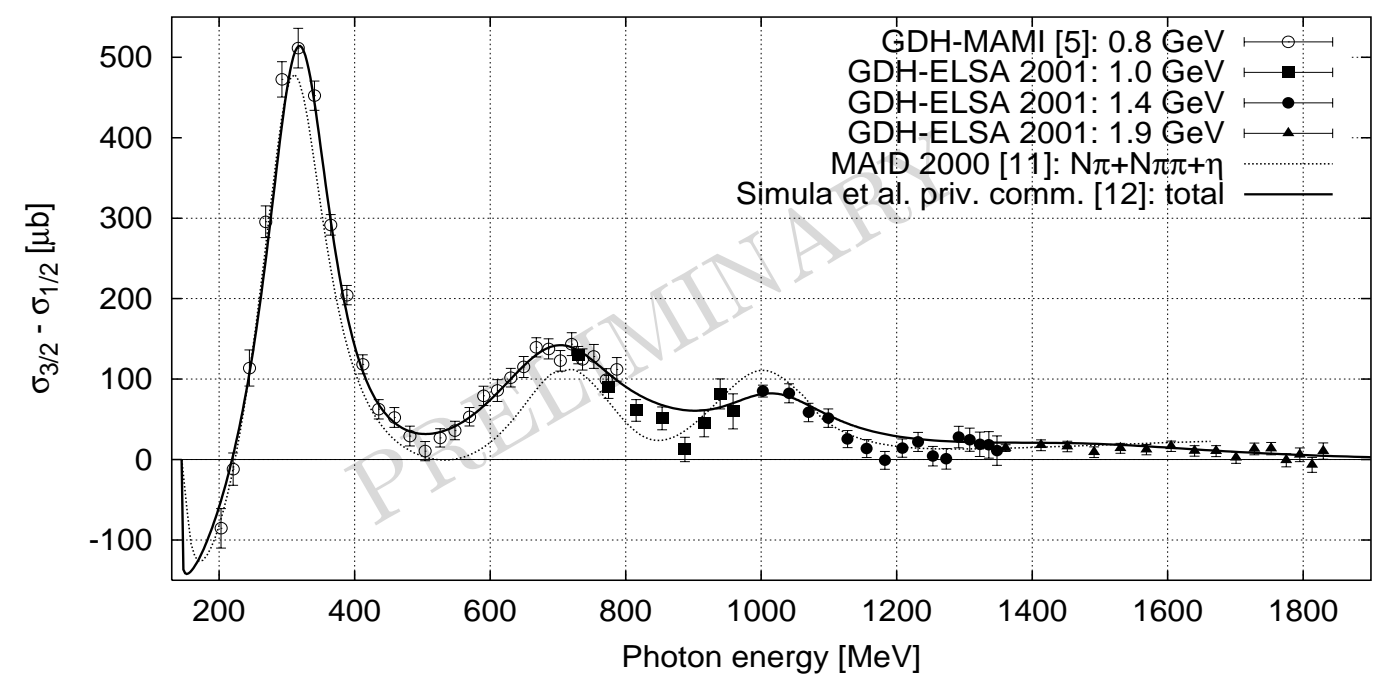

Figure 1: Doubly polarized total cross section difference off the proton compared to models.

the reliability of the whole setup and of the analysis procedure. In addition, we performed several test measurements with polarized beam and polarized target to investigate possible sources of fake asymmetries. All results are compatible with zero.

\section{Results}

Fig. II shows our new preliminary results for the doubly polarized total cross section difference off the proton measured at ELSA at primary electron energies of 1.0, 1.4 and 1.9 $\mathrm{GeV}$. The new data is plotted together with the already published MAMI data $\left[\begin{array}{l}{[\pi} \\ -\end{array}\right]$. The data sets from the different accelerators and from various primary electron energy settings match very well. The $2^{\text {nd }}$ and $3^{\text {rd }}$ resonance are clearly visible and even more pronounced than in the unpolarized hydrogen cross section. The measured difference is positive up to $1.9 \mathrm{GeV}$, which may indicate that the $\mathrm{F}_{35}(1905)$ and $\mathrm{F}_{37}(1950)$ resonances significantly contribute. Our data is compared with two models. The parameterization of the unitary

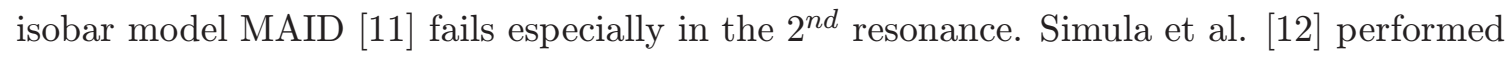
a Regge-based fit to deep inelastic scattering data and extrapolated to $Q^{2}=0$. In addition, they took into account all 4-star-resonances up to $W=2 \mathrm{GeV}$. This model describes our data better, but this is mainly due to an adjustment of the width of the $\mathrm{P}_{33}(1232)$ as well as of the strengths and the widths of the $\mathrm{D}_{13}(1520)$ and $\mathrm{F}_{15}(1680)$ resonances to our data. However, it is remarkable that this model predicts a sign change of $\sigma_{3 / 2}-\sigma_{1 / 2}$ at $2.1 \mathrm{GeV}$.

In Fig.25i-1 we used the measured data to calculate the GDH integral in dependence of the upper integration bound. This so called running GDH integral clearly overruns the sum rule value for the proton of $205 \mu \mathrm{b}$ showing that the question of a sign change is of prime importance. Besides [1] $\left[\begin{array}{l}1 \\ 2\end{array}\right]$ there exists another Regge-inspired analysis of deep inelastic scattering data [i] $\left.{ }_{1}^{1} \overline{3}\right]$. Their parameterization can be extrapolated to $Q^{2}=0$, which gives also a negative sign for $\sigma_{3 / 2}-\sigma_{1 / 2}$ in the Regge regime. Assuming that each Regge prediction [1] 


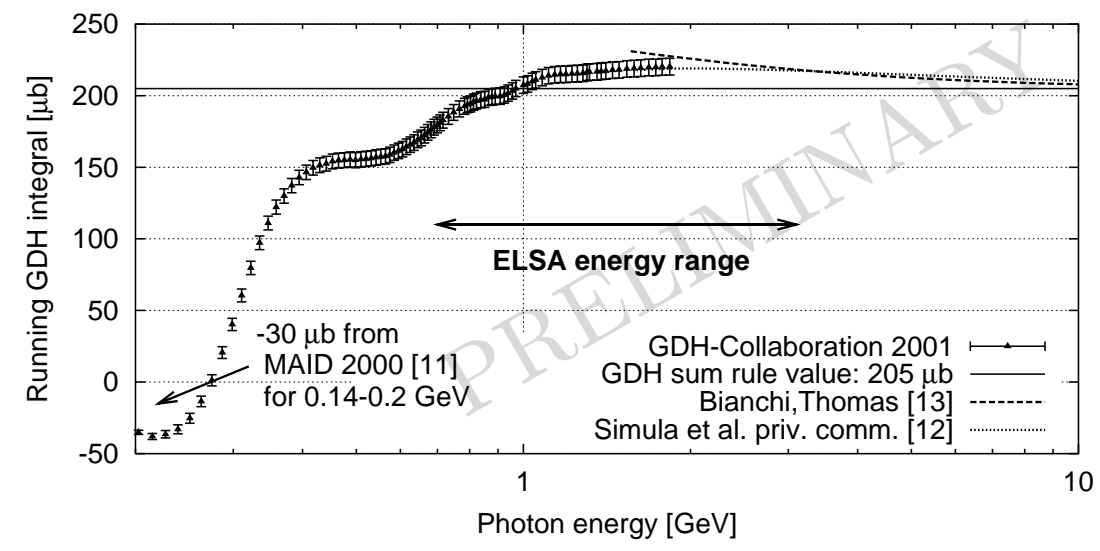

Figure 2: Running GDH integral up to $1.9 \mathrm{GeV}$. Error bars indicate statistical errors only.

development of the running GDH integral as denoted by the dashed and dotted lines in Fig 121 indicating the convergence of the GDH integral. In this figure each predicted high-energy contribution is summed up in dependence of the lower integration bound. As a consequence, these negative contributions would improve the agreement between experimental data and GDH sum rule prediction.

Analysis of the data up to $3.1 \mathrm{GeV}$ will clarify the validity of these Regge predictions in this energy regime and will answer the question whether the GDH sum rule holds. In addition, we will continue the experiment with deuterated butanol at ELSA and MAMI.

\section{References}

[1] Gerasimov, S. B., Sov. Ju. Nucl. Phys. 2 (1966) 430

Drell, S. D. and Hearn, A. C., 'Phys. Rev. Lett. 16 (1966) 908

[2] Low, F. E., 'Phys. Rev. $96-19 \overline{9} 5)$ 1428,

Gell-Mann, M. and Goldberger, M. L., 'Phys.

[3] GDH-Collaboration: Ahrens, J. et al., Phys. Rev. Lett. 84 (2000) 5950 .

[4] GDH-Collaboration: Ahrens, J. et al., 'Phys. Rev. Lett. 87.2001)022003.

[5] Nakamura, S. et al., 'Nucl. Instrum. Meth. 4111 (1998)_93!

[6] Hoffmann, M. et al., SPIN 2000 - Proceedings of the $14^{\text {th }}$ International Spin Physics Symposium, Osaka, Japan, (2001) 756.

[7] Olsen, H. and Maximon, L. C., 'P

[8] Zeitler, G. et al., 'Nucl. Instrum. Meth. $\mathbf{4} \overline{5} \mathbf{9}(2001) 6$.

[9] Helbing, K. et al., accepted, Nucl. Instrum. Meth. A, (2001).

[10] Bradtke, Ch. et al., iNucl. Instrum. Meth. 436 (1999) $430 \overline{1}$

[11] Drechsel, D. et al., "Nucl. Phys.

Drechsel, D. et al., iPhys. Rev.

[12] Simula, S. et al., hep-ph/0107036iand private communication (2001).

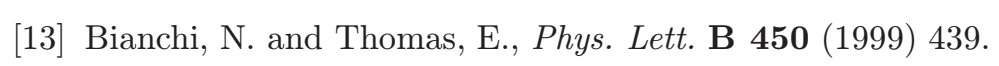

